\title{
Unraveling how Giardia infections cause disease
}

\author{
Theodore E. Nash, MD \\ Gastrointestinal Parasites Section, Laboratory of Parasitic Diseases, National Institute of Allergy and Infectious Diseases, NIH, Bethesda, Maryland, USA.
}

\begin{abstract}
A 40-year-old NIH male scientist camped and fished in a remote lake in Alaska. On his return, he developed diarrhea, cramps, and loose stools without blood or mucus in the absence of fever and was diagnosed with giardiasis. A 3-year-old female living in the Florida Keys complained of intermittent stomachaches over a 2 -month period. Her stools were variably loose. The patient was diagnosed with giardiasis, which led to examination of her mother, father, and brother, who were mildly symptomatic; all 3 were subsequently diagnosed with giardiasis. The child's only exposure was from swimming in a local community pool. A 40-year-old male from Mexico, who resided in Virginia and worked as a cook in a fast food restaurant, was diagnosed with giardiasis. He denied any symptoms and was not allowed to prepare food. Treatment with metronidazole, nitazoxanide, and albendazole failed to eradicate the infection. He was successfully treated with the combination of paromomycin and metronidazole.
\end{abstract}

\section{Current therapy}

In the US, tinidazole, metronidazole, and nitazoxanide are commonly used to treat giardiasis with good, but not perfect, efficacy (1). Quinacrine, which is only available from compounding pharmacies in the US, albendazole, and paromomycin are clinically useful but less often prescribed. Patients who fail single drug regimens, including those with hypogammglobulinemias and AIDS, almost always respond to combination drug treatment (2). In the US and other regions where prevalence is low, disease and epidemics common, and reinfections infrequent, treatment should be offered, but in the third world, where health consequences of infection are unclear and reinfection common, treatment should be reserved for those with likely disease.

\section{Knowledge gap}

As indicated in the sample vignettes, the clinical manifestations and histopathological findings of Giardia infections vary (1). While there are many symptomatic infections and epidemics in the US and similar countries where Giardia infections are sporadic, in highly endemic regions, proven disease due to giardiasis is uncommon and controversial. Published reports have indicated no effects, detrimental effects, and even beneficial effects (3). A

Conflict of interest: The author has declared that no conflict of interest exists.

Citation for this article: J Clin Invest. 2013; 123(6):2346-2347. doi:10.1172/JCI69956. recent meta-analysis suggested that Giardia could be responsible for adverse nutritional effects among children with persistent diarrhea (3).

Although we know there is variability in infection and disease, the cause(s) of this variability are largely unknown. Human experimental infections clearly demonstrated that both host and parasite determinants play a role. Using defined, axenically cultured trophozoites, Nash et al. demonstrated differences in infectivity and virulence among isolates as well as among individuals infected with the same cloned isolate (4). Giardia with identical morphology can be divided into genetic groups $\mathrm{A}$ through $\mathrm{H}$; humans are infected with genetic groups $A$ and $B$, while animals can variably be infected with all genotypes, including genotypes A and B (5). After inoculation with trophozoites of 2 different isolates, the WB isolate belonging to genotype A and the GS isolate of genotype $\mathrm{B}$, none of the volunteers challenged with group A Giardia became infected, while all of the genotype B-challenged individuals became infected and about half developed symptoms (4). At present, the data proving increased virulence of either genotype are inconclusive. The genomes of the prototypical genotype A (WB) and B (GS) isolates vary considerably (6). These differences are also reflected in phenotypical and biological differences. Because certain genotypes are found in both humans and animals, some instances of Giardia infections in humans likely originate from animals; however, except in instances where beavers have contaminated the drinking water (7), convincing point source epidemics from animals to humans have not been recognized.

Dissecting the role of the host's immune response and the role of the parasite is essential to understanding the clinical variability. Many pathological mechanisms have been suggested based on in vitro experiments (8). The judicious use of genetically manipulated Giardia and mice in animal model infections are powerful tools for dissecting the role of the parasite and the host. Similarly, other manipulations of the host, such as inducing malnutrition of the host, as described in the article by Bartelt et al. (9) in this issue of the JCI, can be used with good results.

\section{Research advances}

Bartelt et al. report a variation of a previously described adult mouse infection model that inoculated adult mice using cultured trophozoites of another genotype B Giardia, GS (10). The major modifications from the previous model involve the use of a largely uncharacterized human isolate, $\mathrm{H} 3$, which is maintained by serial passage in animals; the use of cysts purified from animal feces; and infection of malnourished, weaned mice. The major advances of this model are the induction of significant disease over a longer course of infection, which is worsened as a consequence of malnutrition. The latter appears to mimic the circumstances of a sizable proportion of the population in the most highly endemic regions.

Both the H3 and GS isolates are genotype B Giardia, yet infections in adult mice show considerable differences. H3 infection is prolonged, which is more typical of human infections, and induces observable histopathological changes not seen with GS. In the present work and in our previous studies, infection with the prototypical genotype A WB isolate was not sustained in adult mice.

What accounts for these differences between the 2 genotype $\mathrm{B}$ isolates, $\mathrm{H} 3$ and GS? Foremost are the possible genetic differences between the isolates. The $\mathrm{H} 3$ isolate is almost completely unstudied, and nothing is known about its sequence. Two other 
factors could account for the differences in virulence. The $\mathrm{H} 3$ isolate is passaged in animals, most likely gerbils, and cysts excreted in the feces are purified and used to normally initiate infection. In contrast, GS is maintained in culture, which could moderate its virulence over time, and infections are initiated unnaturally by trophozoites. Advantages of the latter are that the inocula are sterile, and this method could control for the expression of varying specific surface proteins (VSPs), which are involved in surface antigenic variation. Specific VSPs may be involved in biological selection and immune escape (11). Furthermore, H3 has not been maintained in culture and would be difficult to genetically manipulate. The interaction of malnutrition and Giardia infection is the most unique aspect of this model and offers a reasonable way to understand the pathophysiology of persistent infection and malnutrition. The presence of eosinophils in the gut and a lack of peripheral eosinophilia are similar to some other gastrointestinal protozoan infections, such as amebiasis, and may play a role in the pathogenesis of disease.

\section{Recommendations}

The presented adult mouse model offers 3 major advantages. The infection is longer and more closely resembles infection and disease in humans. Although not directly compared in this study, infection with the GS isolate is so different in adult mice, that there is a reasonable expectation that what makes a particular Giardia isolate virulent can be dissected using this model. Second, because the immune response is robust, it can be more easily studied. Third, the interaction of malnutrition, which is prominent in many regions where the prevalence of giardiasis is highest, and the manifestations of infection and disease in these regions can be rigorously studied. Even though this model may offer new insight into the pathogenesis of Giardia in the context of malnutrition, it is important to emphasize that there are significant differences between mice and humans.

\section{Acknowledgments}

This commentary was supported by the Intramural Research Program of NIAID, $\mathrm{NIH}$.

Address correspondence to: Theodore E. Nash, Gastrointestinal Parasites Section, Laboratory of Parasitic Diseases, National Institute of Allergy and Infectious Diseases, National Institutes of Health, 9000 Rockville Pike, Building 4, Room 424,
Bethesda, Maryland 20892, USA. Phone: 301.496.6920; Fax: 301.402.0079; E-mail: tnash@niaid.nih.gov.

1. Hill D, Nash TE. Intestinal flagellate and ciliate infections. In: Guerrant R, Walker D, Weller P, eds. Tropical Infectious Diseases: Principles, Pathogens and Practice. 3rd ed. Philadelphia, Pennsylvania, USA: Saunders Elsevier; 2011:623-632.

2. Nash TE, Ohl CA, Thomas E, Subramanian G, Keiser $\mathrm{P}$, Moore TA. Treatment of patients with refractory giardiasis. Clin Infect Dis. 2001;33(1):22-28.

3. Muhsen K, Levine MM. A systematic review and meta-analysis of the association between Giardia lamblia and endemic pediatric diarrhea in developing countries. Clin Infect Dis. 2012; 55(suppl 4):S271-S293.

4. Nash TE, Herrington DA, Losonsky GA, Levine MM. Experimental human infections with Giardia lamblia. J Infect Dis. 1987;156(6):974-984.

5. Cacciò SM, Ryan U. Molecular epidemiology of giardiasis. Mol Biochem Parasitol. 2008;160(2):75-80.

6. Franzen O, et al. Draft genome sequencing of Giardia intestinalis assemblage B isolate GS: is human giardiasis caused by two different species? PLoS Pathogens. 2009;5(8):e1000560.

7. Isaac-Renton JL, Cordeiro C, Sarafis K, Shahriari $\mathrm{H}$. Characterization of Giardia duodenalis isolates from a waterborne outbreak. J Infect Dis. 1993; 167(2):431-440

8. Adam RD. Biology of Giardia lamblia. Clin Microbiol Rev. 2001;14(3):447-475.

9. Bartelt LA, et al. Persistent G. lamblia impairs growth in a murine malnutrition model. J Clin Invest. 2013;123(6):2672-2684

10. Byrd LG, Conrad JT, Nash TE. Giardia lamblia infections in adult mice. Infect Immun. 1994; 62(8):3583-3585.

11. Nash T. Surface antigen variability and variation in Giardia lamblia. Parasitol Today. 1992;8(7):229-234. 